\title{
Rayleigh wave in a micropolar thermoelastic medium without energy dissipation
}

\author{
Baljeet Singh $^{a^{*}}$, Ritu Sindhu ${ }^{\mathrm{b}}$ and Jagdish Singh ${ }^{\mathrm{b}}$
}

${ }^{a}$ Department of Mathematics, Post Graduate Government College, Sector-11, Chandigarh - 160 011, India ${ }^{b}$ Department of Mathematics, M.D.U. Rohtak -124001,Haryana, India

\begin{tabular}{l}
\hline A R T I C L E I N F O \\
\hline Article history: \\
Received 6 April, 2015 \\
Accepted 19 October 2015 \\
Available online \\
19 October 2015 \\
\hline Keywords: \\
Micropolar thermoelasticity \\
Rayleigh wave \\
Frequency equation \\
Speed of propagation \\
\end{tabular}

\section{A B S T R A C T}

\begin{abstract}
The linear governing equations of a micropolar thermoelastic medium without energy dissipation are solved for surface wave solutions. The appropriate solutions satisfying the radiation conditions are applied to the required boundary conditions at the free surface of the half-space of the medium. A frequency equation is obtained for Rayleigh wave in the medium. The non-dimensional speed of the propagation of Rayleigh wave is computed for a specific model of the material and are shown graphically against frequency and non-dimensional parameter.
\end{abstract}

\section{Introduction}

The dynamical theory of thermoelasticity investigates the interaction between thermal and mechanical fields in solid bodies and plays important role in various engineering fields. The generalized theories of thermoelasticity which admit a finite speed of thermal signals (second sound) have aroused much interest during last four decades. For instance, Lord and Shulman (1967), by incorporating a fluxrate term into Fourier's law of heat conduction, formulated a generalized theory which involves a hyperbolic heat transport equation admitting finite speed for thermal signals. Green and Lindsay (1972), by including temperature rate among the constitutive variables, developed a temperature-ratedependent thermoelasticity that does not violate the classical Fourier law of heat conduction, when the body under consideration has a centre of symmetry and this theory also predicts a finite speed for heat propagation. Chandrasekharaiah (1986) referred to this wave-like thermal disturbance as 'second sound'. Green and Naghdi (1977) established a new thermo-mechanical theory of deformable media that uses a general entropy balance as postulated in Green and Naghdi (1991). The theory is explained in detail in the context of flow of heat in a rigid solid, with particular reference to the propagation of themal waves at finite speed. A theory of thermoelasticity for nonpolar bodies, based on the new

* Corresponding author.

E-mail addresses: bsinghgc11@yahoo.com (B. SIngh) 
procedure, was discussed by Green and Naghdi (1993). This theory permits the flow of heat as themal waves at finite speed, and the heat flow does not involve energy dissipation.

The linear theory of micropolar thermoelasticity was developed by extending the theory of micropolar continua to include thermal effects by Eringen (1970) and Nowacki (1986). A generalized theory of linear micropolar thermoelasticity was developed by Boschi and Iesan (1973). Following Green and Lindsay (1972), Dost and Tabarrok (1978) developed a theory of micropolar generalized thermoelasticity. The theory of micropolar thermoelasticity without energy dissipation was developed by Ciarletta (1999), which also admits the finite speed of heat propagation. Singh and Kumar (1998) and Singh (2000) studied some problems on wave propagation in micropolar thermoelasticity in the context of the Lord and Shulman (1967) and Green and Lindsay (1972) theories. Singh (2007) obtained the plane wave solutions of the linear governing equations of a micropolar thermoelastic medium without energy dissipation to show the existence of four plane waves in a two-dimensional model. The expressions for velocities, reflection coefficients, energy ratios of these plane waves are also obtained. The present paper is motivated by the theory of micropolar thermoelasticity formulated by Ciarletta (1999). The surface wave solutions of the linear governing equations for an isotropic micropolar thermoelastic body without energy dissipation are obtained. The particular solutions in the half-space satisfy the radiation conditions and the boundary conditions. The frequency equation of the Rayleigh wave in the half-space is derived. For a particular example of the material, the non-dimensional speed of the Rayleigh wave is computed against the given range of the frequency and non-dimensional parameter.

\section{Governing equations and solution}

A homogeneous, isotropic, micropolar thermoelastic solid occupying the half-space is considered in an undisturbed state, which is initially at uniform temperature $T_{o}$. The rectangular Cartesian coordinates are introduced, having the origin on the surface $\mathrm{z}=0$ and the z-axis is chosen in the direction of increasing depth. A two- dimensional problem (in the $\mathrm{x}$-z-plane) is being discussed with the wave front parallel to the y-axis. Following Green and Naghdi (1993), Eringen (1970) and Ciarletta (1999), the constitutive and the field equations for an isotropic micropolar thermoelastic medium without energy dissipation and in the absence of body forces and couples become

$$
\begin{aligned}
& \sigma_{i j}=\lambda u_{r, r} \delta_{i j}+\mu\left(u_{i, j}+u_{j, i}\right)+\kappa\left(u_{j, i}-\varepsilon_{i j r} \phi_{r}\right)-v \delta_{i j} T, \\
& m_{i j}=\alpha \phi_{r, r} \delta_{i j}+\beta \phi_{i, j}+\gamma \phi_{j, i}, \\
& (\lambda+\mu) \nabla(\nabla . \vec{u})+(\mu+\kappa) \nabla^{2} \vec{u}+\kappa \nabla \times \vec{\phi}-v \nabla T=\rho \ddot{\vec{u}}, \\
& (\alpha+\beta+\gamma) \nabla(\nabla . \vec{\phi})-\gamma \nabla \times(\nabla \times \vec{\phi})+\kappa \nabla \times \vec{u}-2 \kappa \vec{\phi}=\rho j \ddot{\vec{\phi}}, \\
& K^{*} \nabla^{2} T-v T_{0} \nabla \ddot{\vec{u}}=\rho C^{*} \ddot{T},
\end{aligned}
$$

where $\lambda, \mu, \kappa, \alpha, \beta, \gamma$ are material constants, $\rho$ is the density, $j$ is the microinertia,

$v=(3 \lambda+2 \mu+\kappa) \alpha_{t}, \alpha_{t}$ is the coefficient of linear thermal expansion, $C^{*}$ is the specific heat at constant strain and $K^{*}=C^{*}(\lambda+2 \mu) / 4$ is a material constant characteristic of theory. Here $T(x, y, t)$ is the temperature change above the uniform reference temperature $T_{0}, \vec{u}$ is the displacement vector, $\vec{\phi}$ is the microrotation vector, $\sigma_{i j}$ are the components of force stress and $m_{i j}$ are the components of couple stress. The superposed dots denote the time derivatives. For the two-dimensional problem, let

$$
\vec{u}=\left(u_{1}, 0, u_{3}\right) \text { and } \vec{\phi}=\left(0, \phi_{2}, 0\right) \text {, }
$$


where the displacement components $u_{1}$ and $u_{3}$ are written in terms of the potential functions $q(x, y, t)$ and $\psi(x, y, t)$ as

$$
u_{1}=\frac{\partial q}{\partial x}-\frac{\partial \psi}{\partial z}, u_{3}=\frac{\partial q}{\partial z}+\frac{\partial \psi}{\partial x}
$$

and with the help of Eq. (6) and Eq. (7), Eq. (3-5) are reduced to

$$
\begin{aligned}
& (\lambda+2 \mu+\kappa) \nabla^{2} q-v T=\rho \ddot{q}, \\
& K^{*} \nabla^{2} T-v T_{0} \nabla^{2} \ddot{q}=\rho C^{*} \ddot{T}, \\
& (\mu+\kappa) \nabla^{2} \psi-\kappa \phi_{2}=\rho \ddot{\psi}, \\
& \gamma \nabla^{2} \phi_{2}+\kappa \nabla^{2} \psi-2 \kappa \phi_{2}=\rho j \ddot{\phi}_{2}
\end{aligned}
$$

Eq. (8) and Eq. (9) and are coupled in q and T, whereas Eq. (10) and Eq. (11) are coupled in $\phi$ and $\psi$. The solutions of Eq. (8) and Eq. (9) are now sought in the form of the harmonic travelling wave

$$
\{q, T\}=\{\bar{q}(z), \bar{T}(z)\} e^{i k(x-c t)}
$$

Using Eq. (12) in the Eq. (8) and Eq. (9), we obtain two homogeneous equations in $\bar{q}(z), \bar{T}(z)$, which have non-trivial solution if

$$
\begin{aligned}
& K^{*} D^{4}+k^{2}\left(M+L K^{*}+v c^{2} T_{0} Q\right) D^{2}+k^{4}\left(L M-v c^{2} T_{0} Q\right)=0 \\
& \text { where } L=\frac{\rho c^{2}}{\lambda+2 \mu+\kappa}-1, M=\rho c^{2} C^{*}-K^{*}, Q=\frac{v}{\lambda+2 \mu+\kappa} .
\end{aligned}
$$

The general solutions of Eq. (13) are written as

$$
\begin{aligned}
& q=\left(A_{1} e^{-m_{1} z}+A_{2} e^{-m_{2} z}+A_{3} e^{m_{1} z}+A_{4} e^{m_{2} z}\right) e^{i k(x-c t)}, \\
& T=\left(\zeta_{1} A_{1} e^{-m_{1} z}+\zeta_{2} A_{2} e^{-m_{2} z}+\zeta_{1} A_{3} e^{m_{1} z}+\zeta_{2} A_{4} e^{m_{2} z}\right) e^{i k(x-c t)}
\end{aligned}
$$

where

$$
\begin{aligned}
& m_{1}^{2}+m_{2}^{2}=-k^{2}\left[\frac{M+L K^{*}+v c^{2} T_{0} Q}{K^{*}}\right], \quad m_{1}^{2} m_{2}^{2}=k^{4}\left[\frac{L M-v c^{2} T_{0} Q}{K^{*}}\right], \\
& \zeta_{i}=\frac{k^{2}\left[\rho c^{2}+(\lambda+2 \mu+\kappa)\left(\frac{m_{1}^{2}}{k^{2}}-1\right)\right]}{v},(i=1,2) .
\end{aligned}
$$

The solutions of Eq. (10) and Eq. (11) are now sought in the form of the harmonic travelling wave

$$
\left\{\phi_{2}, \psi\right\}=\left\{\bar{\phi}_{2}(z), \bar{\psi}(z)\right\} e^{i k(x-c t)}
$$

Using Eq. (16) in the Eq. (10) and Eq. (11), we obtain two homogeneous equations in $\bar{\phi}_{2}(z), \bar{\psi}(z)$, which have non-trivial solution if 


$$
\gamma D^{4}+k^{2}\left(\gamma P+N+\frac{\kappa R}{k^{2}}\right) D^{2}+k^{4}\left(N P-\frac{\kappa R}{k^{2}}\right)=0,
$$

where $N=\rho j c^{2}-\gamma-\frac{2 \kappa}{k^{2}}, P=\frac{\rho c^{2}}{\mu+\kappa}-1, R=\frac{\kappa}{\mu+\kappa}$.

The general solutions of Eq. (17) are written as

$$
\begin{aligned}
& \phi_{2}=\left(B_{1} e^{-m_{3} z}+B_{2} e^{-m_{4} z}+B_{3} e^{m_{3} z}+B_{4} e^{m_{4} z}\right) e^{i k(x-c t)}, \\
& \psi=\left(\chi_{3} B_{1} e^{-m_{3} z}+\chi_{4} B_{2} e^{-m_{4} z}+\chi_{3} B_{3} e^{m_{3} z}+\chi_{4} B_{4} e^{m_{4} z}\right) e^{i k(x-c t)},
\end{aligned}
$$

where

$$
m_{3}^{2}+m_{4}^{2}=-k^{2}\left[\frac{\gamma P+N+\frac{\kappa R}{k^{2}}}{\gamma}\right], \quad m_{3}^{2} m_{4}^{2}=k^{4}\left[\frac{N P-\frac{\kappa R}{k^{2}}}{\gamma}\right], \chi_{j}=\frac{\left[\rho j c^{2}-2 \frac{\kappa}{k^{2}}+\gamma\left(\frac{m_{j}^{2}}{k^{2}}-1\right)\right]}{\kappa\left(\frac{m_{j}^{2}}{k^{2}}-1\right)},(j=3,4) .
$$

The particular solutions in half-space $(\mathrm{z}>0)$, which satisfy the radiation conditions $q \rightarrow 0, T \rightarrow 0, \phi_{2} \rightarrow 0, \psi \rightarrow 0$ as $z \rightarrow-\infty$ are

$$
\begin{aligned}
& q=\left(A_{1} e^{-m_{1} z}+A_{2} e^{-m_{2} z}\right) e^{i k(x-c t)}, \\
& T=\left(\zeta_{1} A_{1} e^{-m_{1} z}+\zeta_{2} A_{2} e^{-m_{2} z}\right) e^{i k(x-c t)}, \\
& \phi_{2}=\left(B_{1} e^{-m_{3} z}+B_{2} e^{-m_{4} z}\right) e^{i k(x-c t)}, \\
& \psi=\left(\chi_{3} B_{1} e^{-m_{3} z}+\chi_{4} B_{2} e^{-m_{4} z}\right) e^{i k(x-c t)} .
\end{aligned}
$$

\section{Boundary conditions}

The mechanical boundary conditions at $\mathrm{z}=0$ are

$$
\sigma_{z z}=0, \sigma_{z x}=0, \frac{\partial T}{\partial z}=0, m_{y z}=0,
$$

where

$$
\begin{gathered}
\sigma_{z z}=\lambda u_{1,1}+(\lambda+2 \mu+\kappa) u_{3,3}-v T, \\
\sigma_{z x}=\mu u_{3,1}+(\mu+\kappa) u_{1,3}-\kappa \phi_{2}, m_{y z}=\beta \frac{\partial \phi_{2}}{\partial z} .
\end{gathered}
$$

The solutions (20) to (23) satisfy the boundary conditions (24) at $z=0$ and we obtain the following frequency equation

$$
\begin{aligned}
& -a_{1} b_{1} \frac{m_{2}}{k} \frac{m_{4}}{k} \frac{\zeta_{2}}{k^{2}}+a_{1} b_{2} \frac{m_{2}}{k} \frac{m_{3}}{k} \frac{\zeta_{2}}{k^{2}}+a_{2} b_{1} \frac{m_{1}}{k} \frac{m_{4}}{k} \frac{\zeta_{1}}{k^{2}}-a_{2} b_{2} \frac{m_{1}}{k} \frac{m_{3}}{k} \frac{\zeta_{1}}{k^{2}} \\
& -(2 \mu+\kappa)^{2} \frac{m_{1}}{k} \frac{m_{2}}{k} \frac{m_{3}}{k} \frac{m_{4}}{k} \frac{\zeta_{2}}{k^{2}} \chi_{3}+(2 \mu+\kappa)^{2} \frac{m_{1}}{k} \frac{m_{2}}{k} \frac{m_{3}}{k} \frac{m_{4}}{k} \frac{\zeta_{1}}{k^{2}} \chi_{3} \\
& +(2 \mu+\kappa)^{2} \frac{m_{1}}{k} \frac{m_{2}}{k} \frac{m_{3}}{k} \frac{m_{4}}{k} \frac{\zeta_{2}}{k^{2}} \chi_{4}-(2 \mu+\kappa)^{2} \frac{m_{1}}{k} \frac{m_{2}}{k} \frac{m_{3}}{k} \frac{m_{4}}{k} \frac{\zeta_{1}}{k^{2}} \chi_{4}=0,
\end{aligned}
$$


where

$a_{i}=\left[\lambda-(\lambda+2 \mu+\kappa) \frac{m_{i}^{2}}{k^{2}}+v \frac{\zeta_{i}}{k^{2}}\right],(i=1,2)$

$b_{i}=\chi_{k}\left[\mu+(\mu+\kappa) \frac{m_{k}^{2}}{k^{2}}+\frac{\kappa}{k^{2}}\right],(i=1, k=3 ; i=2, k=4)$.

The frequency Eq. (25) reduces for isotropic micropolar case if we take thermal parameter $v=0$. The frequency Eq. (25) can be reduced for isotropic thermoelastic case if we put microrotation parameters $\alpha=\beta=\gamma=\kappa=0$.

\section{Numerical results and discussion}

Following Gauthier (1982), the physical constants for a micropolar thermoelastic solid are considered as

$$
\begin{aligned}
& \lambda=7.59 \times 10^{11} \text { dyne } / \mathrm{cm}^{2}, \mu=1.89 \times 10^{11} \text { dyne } / \mathrm{cm}^{2}, \kappa=0.0149 \times 10^{11} \text { dyne } / \mathrm{cm}^{2}, \\
& \gamma=0.268 \times 10^{11} \text { dyne }, C^{*}=0.23 \mathrm{cal} / \mathrm{g}^{\circ} \mathrm{C}, v=0.05, j=0.0196 \mathrm{~cm}^{2}, \rho=2.19 \mathrm{gm} / \mathrm{cm}^{3}, j k^{2}=0.1
\end{aligned}
$$

With the help of above material constants, the non-dimensional speed $\frac{\rho c^{2}}{\mu+\kappa}$ of the Rayleigh wave is computed for a particular range of the frequency $(\omega)$. The non-dimensional speed of the Rayleigh wave decreases very sharply as we increase the value of frequency in low frequency range, whereas it decreases slowly for higher frequency range. The variation of the non-dimensional speed is shown graphically against the frequency by solid line in Fig. 1, where the dotted line corresponds to the isotropic micropolar case. The comparison of solid and dotted lines in Fig. 1 shows the thermal effects on the speed of Rayleigh wave at different values of frequency.

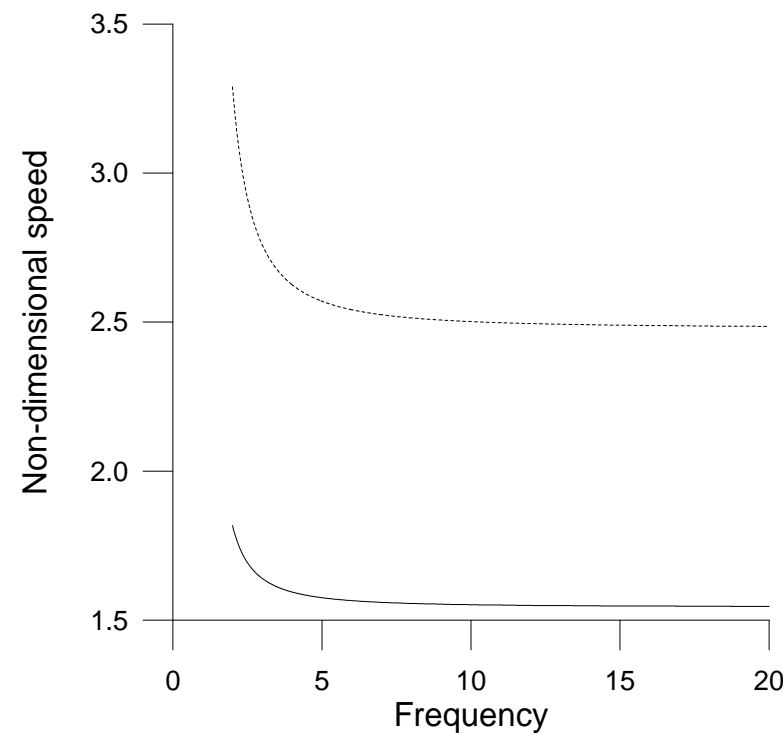

Fig. 1. Variation of the non-dimensional speed $\frac{\rho c^{2}}{\mu+\kappa}$ of the Rayleigh wave against the frequency

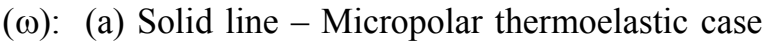

(b) Dotted line - Micropolar elastic case

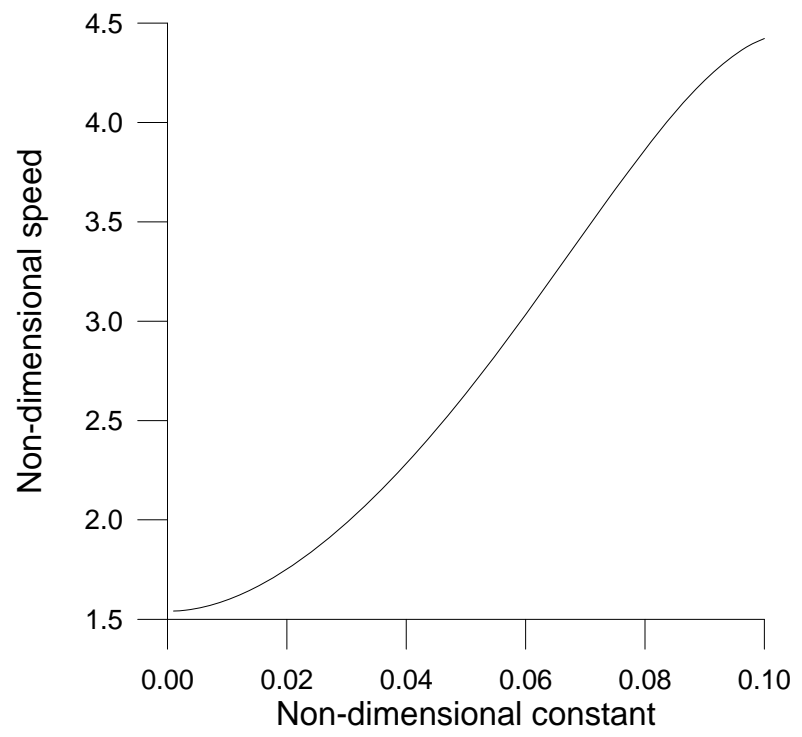

Fig. 2. Variation of the non-dimensional speed $\frac{\rho c^{2}}{\mu+\kappa}$ of the Rayleigh wave against the non-dimensional constant $\frac{v}{\lambda+2 \mu+\kappa}$. 
The non-dimensional speed $\frac{\rho c^{2}}{\mu+\kappa}$ of the Rayleigh wave is also computed against the non-dimensional parameter $\frac{v}{\lambda+2 \mu+\kappa}$. It increases sharply with the increase in value of the non-dimensional constant $\frac{v}{\lambda+2 \mu+\kappa}$. The variation of the non-dimensional speed is shown graphically against the nondimensional constant in Fig. 2.

\section{Conclusion}

The governing equations of the linear, isotropic and homogeneous micropolar thermoelastic medium have been solved for surface wave solutions. The solutions satisfying radiation conditions at the free surface of the half-space are applied to required boundary conditions to obtain the frequency equation. For numerical purpose, the frequency equation of the Rayleigh wave is solved numerically to obtain the values of the non-dimensional speed of the Rayleigh wave for a given range of the frequency and non-dimensional constant. From theory and numerical analysis, it is observed that the non-dimensional speed of the Rayleigh wave depends upon material parameters and the frequency.

\section{References}

Boschi, E., \& Ieşan, D. (1973). A generalized theory of linear micropolar thermoelasticity. Meccanica, $8(3), 154-157$.

Chandrasekharaiah, D. S. (1986). Thermoelasticity with second sound: a review. Applied Mechanics Reviews, 39(3), 355-376.

Ciarletta, M. (1999). A theory of micropolar thermoelasticity without energy dissipation. Journal of Thermal Stresses, 22(6), 581-594.

Dost, S., \& Tabarrok, B. (1978). Generalized micropolar thermoelasticity. International Journal of Engineering Science, 16(3), 173-183.

Gauthier, R. D. (1982). Experimental investigations on micropolar media. Mechanics of Micropolar Media, 395-463.

Green, A. E., \& Lindsay, K. A. (1972). Thermoelasticity. Journal of Elasticity, 2(1), 1-7.

Green, A. E., \& Naghdi, P. M. (1977, November). On thermodynamics and the nature of the second law. In Proceedings of the Royal Society of London A: Mathematical, Physical and Engineering Sciences (Vol. 357, No. 1690, pp. 253-270). The Royal Society.

Green, A. E., \& Naghdi, P. M. (1991, February). A re-examination of the basic postulates of thermomechanics. In Proceedings of the Royal Society of London A: Mathematical, Physical and Engineering Sciences (Vol. 432, No. 1885, pp. 171-194). The Royal Society.

Green, A. E., \& Naghdi, P. M. (1993). Thermoelasticity without energy dissipation. Journal of Elasticity, 31(3), 189-208.

Eringen, A. C. (1970). Foundations of micropolar thermoelasticity. Berlin: Springer.

Lord, H. W., \& Shulman, Y. (1967). A generalized dynamical theory of thermoelasticity. Journal of the Mechanics and Physics of Solids, 15(5), 299-309.

Nowatzki, W. (1986). Theory of asymmetric elasticity. Oxford: Pergamon.

Singh, B., \& Kumar, R. (1998). Reflection of plane waves from the flat boundary of a micropolar generalized thermoelastic half-space. International Journal of Engineering Science, 36(7), 865-890.

Singh, B. (2000). Reflection of plane sound wave from a micropolar generalized thermoelastic solid half-space. Journal of Sound and Vibration, 235(4), 685-696.

Singh, B. (2007). Reflection coefficients and energy ratios in a micropolar thermoelastic medium without energy dissipation. The ANZIAM Journal, 48(03), 433-447. 